\title{
Correspondence
}

\section{Parenteral nutrition compared with transpyloric feeding}

Sir,

Glass et al ${ }^{1}$ compared parenteral nutrition with transpyloric feeding in two matched groups of infants weighing less than $1500 \mathrm{~g}$ at birth. In their hands parenteral nutrition resulted in a $40 \%$ mortality rate and a $70 \%$ infection rate. They recommend a method of transpyloric feeding which had to be abandoned in one third of their patients in favour of parenteral nutrition, while one third of the remainder developed necrotizing enterocolitis.

We feel that this study is based on the false premise that there is one optimal regimen of feeding which can be rigidly administered to all low birthweight infants irrespective of their clinical status. Such an approach led to unacceptably high mortality and morbidity which raises serious ethical questions. We were surprised that they continued with it for a period of one year in the face of such disturbing results.

We contend that a flexible approach based on a full clinical assessment of the individual patient would avoid many of the difficulties encountered by the authors. It is our practice to introduce enteral feeds only after stabilising the vital signs of the infant and establishing acid base and biochemical homeostasis. It is important to increase the volume of milk according to a continuous assessment of the individual infant's tolerance judged by gastric retention, the presence of bile stained aspirate, abdominal distention or tenderness, and the passing of normal motions. The full nutritional needs of the individual infant may be met by supplementing enteral feeds by partial parenteral nutrition until full enteral feeding is established. By the same process of reasoning we agree with the authors that a rigid policy of withholding enteral feeds in the first two to three weeks of life in all very low birthweight infants as suggested by Eyal et al ${ }^{2}$ is unnecessary but support their plea for caution in the introduction of milk.

In a small proportion of very immature or sick infants total parenteral nutrition may be required for prolonged periods because they are unable to tolerate enteral feeds based on the above criteria. Contrary to the experience of Glass et $a l^{1}$ we have found this to be a safe and effective procedure when properly managed in agreement with the findings of Yu et al. ${ }^{3}$

By adopting this individualised flexible approach based on continuous clinical and laboratory monitoring, the iatrogenic infection rate of our very low birthweight infants is less than three per cent and the incidence of necrotizing enterocolitis is less than two per cent. We expect a survival rate of over $95 \%$ for infants between 1 and $1.5 \mathrm{~kg}$ and of over $60 \%$ in infants under $1 \mathrm{~kg}$, excluding those with lethal congenital malformations

S D Ferguson, M D Yohannan, and K I Blake Royal Gwent Hospital, Newport,
Dr Glass and co-authors comment:

Dr Ferguson and his colleagues have failed to comprehend our paper.' We did not 'recommend' a method of transpyloric feeding but adopted a method deemed appropriate on the basis of the published reports. This study was based on a premise which is anything but false, namely that the different methods of feeding which are advocated for the low birthweight infant need to be looked at critically and assessed objectively by proper clinical trials if we are to understand how best to meet the nutritional needs of these infants.

This trial was indicated because the results with the 'flexible' type of approach which Dr Ferguson and his colleagues advocated proved so unsatisfactory. 'Full clinical assessment' and, as far as possible, 'stabilising the vital signs of the infant and establishing acid base and biochemical homeostasis' are practised on all infants in this unit but do not of themselves answer adequately the question as to the best methods of feeding low birthweight infants. Properly conducted clinical trials using accepted methods of feeding are an essential element in answering this question adequately and the results must be reported no matter how unsatisfactory they prove to be.

In such trials it is the comparison between different methods which is important. As this unit is a regional one accepting low birthweight infants referred from other hospitals, the proportion of high risk low birthweight infants for whom it cares is high. As we pointed out in our paper ${ }^{1}$ most infants in the trial who died in the first week did so from intraventricular haemorrhage, and it is doubtful whether the method of feeding played any part in their aetiology or whether any 'flexibility' other than the carefully considered switching to an alternative method of feeding which took place in our trial when one method proved unsuitable would have made any difference. The claims of Dr Ferguson and his colleagues would have been more credible and responsible if they had quoted an actual rather than an expected mortality rate and had defined their population and their criteria.

Dr Ferguson and his colleagues also quote a meaningless infection rate based on an 'iatrogenic infection rate' (whatever that is) and the exclusion of infants with lethal congenital malformations. Our infection rate was uninfluenced by such dubious qualifications.

The Archives of Disease in Childhood rightly demands high ethical standards and invites those whose opinions it seeks as referees to pronounce on the ethics of any paper submitted for publication. A sinister danger to the welfare of children, however, would be a reluctance by those who have conducted careful and ethical research to publish unsatisfactory results honestly and frankly lest some whose practices differ or whose prejudices blind should seek too readily to discredit this essential research not by scientific data or logical argument but by irresponsible, ill considered imputations of unethical conduct.

\section{References \\ ${ }^{1}$ Glass EJ, Hume R, Lang MA, Forfar JO. Parenteral nutrition}


compared with transpyloric feeding. Arch Dis Child 1984;59:131-5.

${ }^{2}$ Eyal F, Sagi E, Arad I, Auital A. Necrotising enterocolitis in the very low birthweight infant: expressed breast milk feeding compared with parenteral feeding. Arch Dis Child 1982;57: 274-6.

3 Yu UYH, James B, Hendry P, MacMahon RA. Total parenteral nutrition in very low birthweight infants: a controlled trial. Arch Dis Child 1979;54:653-61.

\section{Oral rehydration fluids}

Sir,

Tripp and Candy have reviewed the present state of oral rehydration clearly and concisely. ${ }^{1}$ I would like to make a few comments.

In most places where the World Health Organisation (WHO) oral rehydration solution is used a large proportion of patients will have varying degrees of malnutrition. Thus, experience of treatment in developing countries is not necessarily applicable to developed countries where intravenous fluids are available. In developing countries most children who die from dehydration die because they have not received intravenous fluids appropriately, or at all. The purpose of oral rehydration solution is to prevent them getting to this stage. Although WHO oral rehydration solution can be used successfully, even in severely dehydrated children if carefully managed, it does not negate the necessity to have facilities for intravenous treatment. About 10 to $15 \%$ of children in hospital treated with oral rehydration solution will need the addition of intravenous fluids to achieve rehydration. ${ }^{2}$ Unfortunately many children with moderate to severe dehydration die when given oral rehydration solution because it is given unsupervised resulting in progression of dehydration, and intravenous fluids are not given or are given too late.

In developed countries most children with diarrhoea who are not dehydrated or only mildly so are adequately treated with a low osmolar solution, and those more severely dehydrated with intravenous fluids. A $100 \%$ cure rate is the aim. I do not see why, unless the health facilities deteriorate in these countries, the practice should be changed. In fact rehydrating a moderately dehydrated child with oral rehydration solution is much more time consuming for nursing staff than administration of intravenous fluids with the aid of an infusion pump.

Oedema in children given oral rehydration solution may be a symptom of malnutrition rather than of overhydration. Many malnourished children develop oedema for the first time after rehydration with the WHO oral rehydration solution. This does not necessarily mean that they are adequately rehydrated but more that the sodium load of oral rehydration solution is too much for their already compromised state, which includes a reduced plasma oncotic pressure and impaired renal tubular function.

It is suggested that the potassium concentration of the WHO oral rehydration solution is too low to replace the total body potassium deficit that is commonly found in malnourished children with diarrhoea. What is more important than increasing the potassium concentration of the present solution, which ideally is only used for rehydration and not maintenance, is to give potassium supplements for some days or weeks after rehydration in the form of potassium salts, bananas, coconut water, or citrus fluids. In the case of the occasional child who presents with symptoms of hypokalaemia such as hypotonia, abdominal distention, and ileus only high doses of intravenous potassium followed by an oral potassium for two to three weeks will suffice.

Regarding appropriate technology, it is important to separate the children with nil to mild dehydration who can be adequately treated with a variety of fluids from the moderate to severely dehydrated child who in the absence of intravenous treatment can only be rehydrated by a carefully managed high osmolar solution. The osmolality of 'home based solutions' can vary enormously. A low osmolar solution will not be adequate for the treatment of these children.

Lastly, it has been stated that neonates can be successfully rehydrated by the WHO oral rehydration solution. The physiology of the neonate in developing countries is no different to that in developed countries where solutions containing $0 \cdot 18 \mathrm{~N}$ saline are used for rehydrating neonates. At present, in view of the neonates' limited ability to excrete sodium, it would seem unwise to advocate the universal use of WHO oral rehydration solution for management of dehydration in neonates until it has been shown that it is safe when given unsupervised. Unfortunately, with the number of dehydrated infants presenting to health centres in developing countries, supervision of rehydration is the exception rather than the rule.

J B S Coulter Liverpool School of Tropical Medicine, Pembroke Place, Liverpool L3 $5 Q A$

Sir,

Drinks will not help a child in circulatory collapse from diarrhoea; liquid by vein will.

Unless enthusiasts for oral rehydration make this clear children may die and an old and valued remedy for moderate or early enteritis will be blamed. Drs Tripp and Candy in their annotation ${ }^{1}$ should surely have reminded us that in the paper by Santosham et al, ${ }^{3}$ on which they rely heavily, all children with severe diarrhoea (shock or $10 \%$ dehydration) received intravenous treatment before entering the trial.

$\mathrm{T}$ H Hughes-Davies 6 Tavistock Court, Tavistock Square, London WC1

Drs Tripp and Candy comment:

One of the reasons for writing our annotation ${ }^{1}$ was to review the controversies and stimulate discussion on the subject of oral rehydration treatment for acute diarrhoea. We were therefore grateful to receive letters from Drs Hughes-Davies and Coulter and for this opportunity to comment. Both correspondents have highlighted the need 